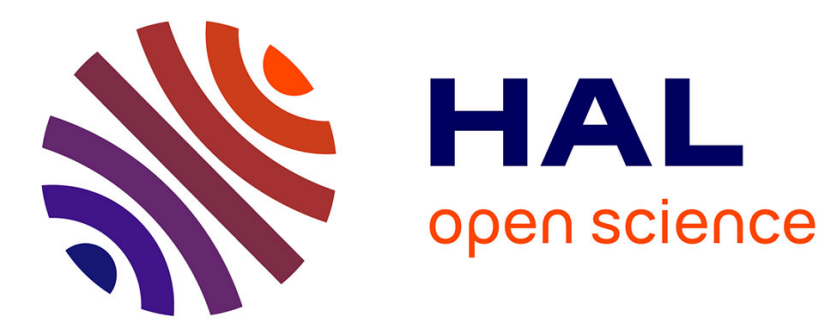

\title{
Seismiclike organization of avalanches in a driven long-range elastic string as a paradigm of brittle cracks
}

\author{
Jonathan Bares, Daniel Bonamy, Alberto Rosso
}

\section{To cite this version:}

Jonathan Bares, Daniel Bonamy, Alberto Rosso. Seismiclike organization of avalanches in a driven long-range elastic string as a paradigm of brittle cracks. Physical Review E , 2019, 100 (2), pp.023001. 10.1103/PhysRevE.100.023001 . hal-02269109

\section{HAL Id: hal-02269109 \\ https://hal.science/hal-02269109}

Submitted on 22 Aug 2019

HAL is a multi-disciplinary open access archive for the deposit and dissemination of scientific research documents, whether they are published or not. The documents may come from teaching and research institutions in France or abroad, or from public or private research centers.
L'archive ouverte pluridisciplinaire HAL, est destinée au dépôt et à la diffusion de documents scientifiques de niveau recherche, publiés ou non, émanant des établissements d'enseignement et de recherche français ou étrangers, des laboratoires publics ou privés. 


\title{
Seismiclike organization of avalanches in a driven long-range elastic string as a paradigm of brittle cracks
}

\author{
Jonathan Barés, ${ }^{1}$ Daniel Bonamy, ${ }^{2}$ and Alberto Rosso ${ }^{3}$ \\ ${ }^{1}$ Laboratoire de Mécanique et Génie Civil, Université de Montpellier, CNRS, Montpellier, France \\ ${ }^{2}$ SPEC/SPHYNX, DSM/IRAMIS CEA Saclay, Bat. 772, F-91191 Gif-sur-Yvette, France \\ ${ }^{3}$ LPTMS, CNRS, Univ. Paris-Sud, Université Paris-Saclay, 91405 Orsay, France
}

(Received 10 May 2019; published 1 August 2019)

\begin{abstract}
Crack growth in heterogeneous materials sometimes exhibits crackling dynamics, made of successive impulselike events with specific scale-invariant time and size organization reminiscent of earthquakes. Here, we examine this dynamics in a model which identifies the crack front with a long-range elastic line driven in a random potential. We demonstrate that, under some circumstances, fracture grows intermittently, via scale-free impulse organized into aftershock sequences obeying the fundamental laws of statistical seismology. We examine the effects of the driving rate and system overall stiffness (unloading factor) onto the scaling exponents and cutoffs associated with the time and size organization. We unravel the specific conditions required to observe a seismiclike organization in the crack propagation problem. Beyond failure problems, implications of these results to other crackling systems are finally discussed.
\end{abstract}

DOI: 10.1103/PhysRevE.100.023001

\section{INTRODUCTION}

Crackling systems encompass a broad range of systems; those that, under slowly varying external forcing, respond via series of violent random impulses, so-called avalanches. Crack growth [1-4], damage spreading in compressed solids [5-10], Portevin-Le Chatelier effect in alloys [11-13], plastic deformation in crystalline [14-17], noncrystalline [18] and divided solids [19], magnetization change in ferromagnets [20-22], imbibition in porous media [23-26], earthquakes [27-30], neuronal activity [31,32], strain in shapememory alloys [33], magnetic vortex dynamics in superconductor $[34,35]$, etc., are illustrative examples of crackling noise. A key feature in these systems is that the individual avalanches exhibit universal scale-free statistics and scaling laws, independent of the microscopic and macroscopic details but fully set by generic properties such as symmetries, dimensionality, and interaction range (see [36] for review). In some of these systems, these scale-free features can be understood in the framework of the depinning transition of elastic manifolds, separating a quiescent phase where the system is trapped by the landscape disorder and an active phase where the applied forcing is sufficient to make the manifold escape from all metastable states and evolve at finite speed [37,38]. Functional renormalization theory (FRG) then provides the relevant framework to describe the observed features [39-43].

Beyond the specific scale-free features obeyed by individual avalanches, crackling systems sometimes display temporal correlations. This is, e.g., manifested by power-law distributed waiting times between successive events $[6,8,10,27,44,45]$. Another illustrative example is found in seismology; earthquakes get organized into aftershock $(A S)$ sequences which obey characteristic laws [46]: productivity law [47,48] stating that the number of produced aftershocks goes as a power law with the main shock $(M S)$ energy, Båth's law [49] stipulating that the ratio between the $M S$ energy and that of its largest $A S$ is independent of the $M S$ magnitude, and Omori-Utsu law [50-52] telling that the production rate of $A S$ decays algebraically with the time elapsed since $M S$. These laws, referred to as the fundamental laws of seismology, are central in the implementation of probabilistic forecasting models of earthquakes [53]. They are not specific to seismology and were also reported, at the laboratory scale, in the acoustic emission associated with the damaging of different materials loaded under compression [8,9], in the global dynamics of a sheared granular material $[19,54]$ and in the simpler situation of a single tensile crack slowly driven in artificial rocks [45]. In the latter case, it has been possible to show that the fundamental laws of seismology are direct consequences of the individual scale-free statistics of both the event sizes and interevent waiting times [45,55]; productivity and Båth's law [49] for $A S$ sequences result from the power-law distribution of sizes and Omori-Utsu law results from the power-law distribution of waiting time.

Noticeably, the simplest (and standard) picture of elastic manifolds driven quasistatically in a random potential fails to reproduce the above time clustering features [56]. Those can be recovered by adding supplementary ingredients, such as, e.g., memory effects [6], viscoelasticity [57], other slow relaxation processes $[15,58,59]$, or a finite temperature [60]. A more general explanation has been proposed in [44,61-63]: power-law distributed interevent waiting times simply arise when a finite detection threshold is applied to separate the events from the background noise. As driving rates are finite in experiments and, hence, finite detection thresholds are required, this argument combined with the fact scalefree statistics for both sizes and waiting times directly yield aftershock sequences and seismic laws [45,55] provides an explanation of the seismiclike temporal organization widely 
reported in damage and fracture problems. Still, the specific conditions leading to this organization remain to be clarified.

We report here a theoretical and numerical study of the fracture problem in its most fundamental state: a single propagating crack growing throughout an elastic heterogeneous material. This problem is classically identified with the motion of a one-dimensional (1D) long-range elastic string moving in an effective two-dimensional (2D) random media [64-67]; the different steps underpinning the description are summarized in Sec. II. For some conditions, this motion displays a crackling dynamics, made of successive avalanches obeying the fundamental laws of seismicity (Sec. III). The specific conditions required to observe the seismiclike organization of successive events are finally discussed (Sec. IV).

\section{THEORETICAL AND SIMULATION FRAMEWORK}

The existence of cracks in solids dramatically amplifies applied stresses in their vicinity. This mechanism makes the fracture behavior at the macroscopic scale extremely sensitive to the presence of defects and/or microcracks down to very small scales, which translates into large statistical aspects difficult to assess in practice. For brittle solids under tension, the difficulty is sidetracked by reducing the problem to the destabilization of a single preexisting crack in an otherwise intact material (see [68] for a recent review). Strength statistics and its size dependence are analyzed within the Weibull weakest-link framework [69] and linear elastic fracture mechanics (LEFM) provides the theoretical framework to describe crack destabilization and further growth (see, e.g., Ref. [70]).

\section{A. Crack growth in homogeneous materials: Continuum fracture mechanics}

Let us consider the situation depicted in Fig. 1(a) of a crack front propagating in a brittle solid embedding microstructural inhomogeneities, loaded by applying tensile stresses $\sigma_{0}$ (or by imposing a displacement field $u_{0}$ ) along its external surfaces. In the following section, we present the fundamental concepts of fracture mechanics and we adopt the usual conventions of this field: the axes $x, y$, and $z$ align with the mean direction of crack propagation, tensile loading, and mean crack front. Moreover, $L$ denotes the specimen thickness along $z$. Continuum engineering mechanics simplifies the problem by (i) coarse-graining the solid into an effective linear elastic homogeneous material of Young modulus $E$ [70], (ii) considering a straight crack, without any roughness, and (iii) averaging the behavior along $z$ to reduce the $3 \mathrm{D}$ elastic problem to a $2 \mathrm{D}$ one.

The question of when the crack starts growing is then solved by looking at how the total energy evolves with the crack length, $f$. In a perfectly brittle material, this total energy evolves due to two contributions: the potential elastic energy, $\Pi_{\text {pot }}$, stored in the pulled solid and the energy dissipated to create the crack surfaces, $\Pi_{\text {surf }}$. The former decreases with $f$; in the limit of plates with large $x$ and $y$ dimensions, $\Pi_{\text {pot }}(f) \approx$ $\Pi_{\text {pot }}(f=0)-\sigma_{0}^{2} L f^{2} / E$. The latter increases linearly with $f: \Pi_{\text {surf }}=\Gamma L f$, where $\Gamma$ is the fracture energy. When $\sigma_{0}$ is small, the evolution of the total energy with $f$ is dominated

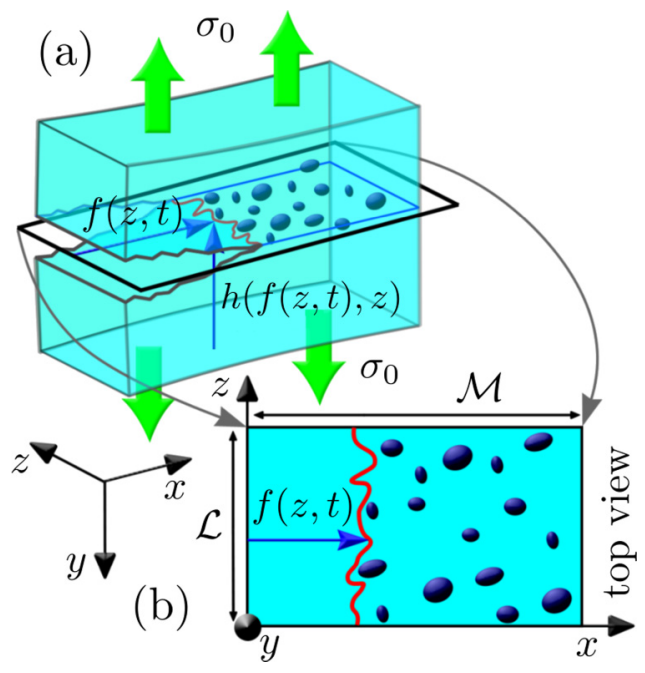

FIG. 1. Schematic view of a single crack growing in a perfectly brittle heterogeneous material. (a) 3D view of the crack propagating from left to right, opened by the stress $\sigma_{0}$. The crack front shape (red line) is described horizontally by the function $f(z, t)$ and vertically by the function $h(f(z, t), z)$. (b) 2D projection on the mean crack plane $(x, z)$. The sample length is $\mathcal{M}$, while its periodic width is $\mathcal{L}$. Ellipses stand for heterogeneities. See the text for more details.

by the increase of $\Pi_{\text {surf }}$ and the crack is stable. When $\sigma_{0}$ is large, $\Pi_{\text {tot }}$ is dominated by $\Pi_{\text {pot }}$ which decreases with $f$ and hence the crack propagates. Griffith introduces the energy release rate, $G$, defined as $G=-(1 / L)\left(d \Pi_{\text {pot }} / d f\right)$, which is the amount of energy released as $f$ increases of a unit step $[70,71]$ and the propagation criterion is

$$
G>\Gamma
$$

where, in the limit of plates of large $x$ and $y$ dimensions, $G \approx$ $\sigma_{0}^{2} f / E$ and more generally

$$
G=\frac{\sigma_{0}^{2} f}{E} \mathcal{F}\left(f / L_{i}, L_{j} / L_{i}\right),
$$

where $\mathcal{F}\left(f / L_{i}, L_{j} / L_{i}\right)$ is a dimensionless function of the various macroscopic lengths $L_{i}$ invoked to describe the geometry: the specimen dimensions $L_{x}$ and $L_{y}$, the position of the crack, of the loading points, etc.

Once the crack starts propagating, an additional contribution due to kinetic energy, $\Pi_{\text {kin }}$, is to be taken into account in the total system energy. The crack speed, $v=\dot{f}(t)$, is then selected so that the total elastodynamics energy released as the crack propagates over a unit length exactly balances the fracture energy: $G^{\text {dyn }}(v)=-(1 / v) d\left(\Pi_{\text {pot }}+\Pi_{\text {kin }}\right) / d t=$ $\Gamma$. Assuming that the specimen is large enough so that the elastic waves emitted by the propagating crack cannot reflect on the boundaries and come back to perturb the crack motion, this equation reduces to [72]

$$
A(v) G=\Gamma, \quad \text { with } A(v) \approx 1-\frac{v}{c_{R}},
$$

where $c_{R}$ is the Rayleigh wave speed. For a slow enough motion, Eq. (3) reduces to

$$
\frac{1}{\mu} v=G-\Gamma,
$$

where the effective mobility $\mu$ is given by $\mu=c_{R} / \Gamma$. 
It is worth noting that any situation where the solid is loaded by imposing the external stress breaks in a brutal manner. Indeed, $G$ increases with $f$ [Eq. (2)]. This means that as soon as the crack starts growing, $G$ increases, making $v$ increase all the more rapidly, etc. Conversely, situations involving a loading by a constant displacement rate, $\dot{u}_{0}$, may yield stable crack growth. Indeed, $\sigma_{0}=k(f) u_{0}(t)$, where the system stiffness $k(f)$ is always decreasing with crack length. Equation (2) becomes

$$
G(f, t)=\frac{\dot{u}_{0}^{2} t^{2} k(f) f}{E} \mathcal{F}\left(f / L_{i}, L_{j} / L_{i}\right) .
$$

In some situations, the above expression yields $G$ decreasing with increasing $f$. Then, the crack propagates in a stable manner, so that $G$ remains always close to $\Gamma$. Without loss of generality, we choose a reference time $t_{0}$ and crack length $f_{0}$ so that $G\left(f_{0}, t_{0}\right)=\Gamma$ (right at propagation onset) and look at the crack dynamics in the vicinity of this reference after having shifted the origin: $f \rightarrow f-f_{0}$ and $t \rightarrow t-t_{0}$. Equation (4) writes

$$
\frac{1}{\mu} \frac{d f}{d t}=\dot{G} t-G^{\prime} f
$$

where $\dot{G}=\partial G /\left.\partial t\right|_{\left\{t_{0}, f_{0}\right\}} \quad$ (driving rate) and $G^{\prime}=$ $-\partial G /\left.\partial f\right|_{\left\{t_{0}, f_{0}\right\}}$ (unloading factor) are positive constants. In this stable configuration, the crack first displays a transient and then grows at a constant speed $v=\dot{G} / G^{\prime}$.

\section{B. Crack growth in heterogeneous materials: Depinning line model of cracks}

Equation (6) predicts continuous dynamics in stable crack growth situations, in contradiction with the crackling dynamics sometimes observed in experiments $[1,4]$. The depinning approach $[64,66,67]$ consists in taking into account the microstructure inhomogeneities by adding a stochastic term in the local fracture energy: $\Gamma(x, y, z)=\bar{\Gamma}+\gamma(x, y, z)$. This induces in-plane $[f(z, t)]$ and out-of-plane $[h(f(z, t), t)]$ distortions of the front [Fig. 1(a)] which, in turn, generate local variations in $G$. To the first order, the variations of $G$ depend on the in-plane front distortion only [Fig. 1(b)] and the problem reduces to that of a planar crack $[h(f(z, t), t)=$ const] [73]. One can then use Rice's analysis [74,75] to relate the local value $G(z, t)$ of energy release to the front shape, $f(z, t)$ [Fig. 1(b)]:

$$
\begin{aligned}
G(z, t) & =\bar{G}(\bar{f}, t)[1+J(z,\{f\})], \\
\text { with } J(z,\{f\}) & =\frac{1}{\pi} P V \int_{\text {crack front } f} \frac{f(\zeta, t)-f(z, t)}{(\zeta-z)^{2}} d \zeta,
\end{aligned}
$$

where $P V$ denotes the principal part of the integral; the longrange kernel $J$ is more conveniently defined by its $z$-Fourier transform $\hat{J}(q)=-|q| \hat{f} . \bar{G}(\bar{f}, t)$ denotes the energy release rate that would have been used in the standard continuum picture, after having coarse-grained the microstructure disorder and having replaced the distorted front by a straight one at the mean position $\bar{f}(t)$ (averaged over the specimen thickness). The application of Eq. (6) at each point $z$ of the crack front supplemented by Eq. (7) yields

$$
\frac{1}{\mu} \frac{\partial f}{\partial t}=\dot{G} t-G^{\prime} \bar{f}+\bar{\Gamma} J(z,\{f\})+\gamma[z, x=f(z, t)] .
$$

The random term $\gamma(z, x)$ is characterized by two main quantities: the noise amplitude defined as $\tilde{\Gamma}=\left\langle\gamma^{2}(x, z)\right\rangle_{x, z}^{1 / 2}$ and the spatial correlation length $\ell$ over which the correlation function $C(\vec{r})=\left\langle\gamma\left(\vec{r}_{0}+\vec{r}\right) \gamma\left(\vec{r}_{0}\right)\right\rangle_{\vec{r}_{0}}$ decreases [67].

Equation (8) provides the equation of motion of the crack line. A priori, it involves seven parameters: $\mu, \bar{\Gamma}, \dot{G}, G^{\prime}$, $\ell, \tilde{\Gamma}$, and the specimen thickness $\mathcal{L}$. The introduction of dimensionless time, $t \rightarrow t /(\ell / \mu \bar{\Gamma})$, and space, $\{x, z, f\} \rightarrow$ $\{x / \ell, z / \ell, f / \ell\}$, allows reducing this number of parameters to four. The resulting equation of motion writes

$$
\frac{\partial f}{\partial t}=c t-k \bar{f}+J(z,\{f\})+\gamma(z, f(z, t)),
$$

where $c=\dot{G} \ell / \mu \bar{\Gamma}^{2}$ is the dimensionless loading speed and $k=G^{\prime} \ell / \bar{\Gamma}$ is the dimensionless unloading factor. The two other parameters are the dimensionless system size $N \rightarrow \mathcal{L} / \ell$ and the dimensionless noise amplitude $\tilde{\Gamma} \rightarrow \tilde{\Gamma} / \bar{\Gamma}$.

\section{Numerical methods, avalanche detection, and sequence identification}

In the following, both system size and noise amplitude are constant: $N=1024$ and $\tilde{\Gamma}=1$. The line is discretized along $z: f(z, t)=f_{z}(t)$ with $z=1, \ldots, N$ and the time evolution of $f_{z}(t)$ is obtained by solving Eq. (9) using a fourth order Runge-Kutta scheme, as in [67,76]. The second right-hand term in Eq. (9) is obtained using a discrete Fourier transform along $z$ (periodic conditions along $z$ ). A discrete uncorrelated random Gaussian matrix $\gamma_{z, x}$ is prescribed (zero average and unit variance). The third right-hand term in Eq. (9) is obtained

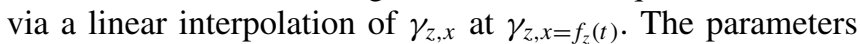
$c$ and $k$ in the first right-handed term of Eq. (9) are varied from $10^{-6}$ to $5 \times 10^{-4}$ and from $10^{-4}$ to 0.5 , respectively. The movie provided as Supplemental Material [77] illustrates the jerky motion of the crack front (in red) obtained via these simulations.

The crackling noise signal considered in the following is the instantaneous, spatially averaged, crack speed:

$$
\bar{v}(t)=\frac{1}{N} \sum_{z=1}^{N} \frac{d f_{z}}{d t} .
$$

An example of such signal is shown in Fig. 2(a). The avalanches are then identified with the bursts of $\bar{v}(t)$ above a prescribed threshold $v_{t h}$; an avalanche $i$ starts at $t_{i}^{\text {start }}=t_{i}$ when the signal rises above $v_{t h}$ and ends at $t_{i}^{\text {end }}$ when $\bar{v}(t)$ goes back below this value. The size is then defined by $S_{i}=$ $N \int_{t_{i}^{\text {start }}}^{t_{\text {end }}^{\text {end }}}\left[\bar{v}(t)-v_{t h}\right] d t$ and the interevent waiting time between avalanches $i$ and $i+1$ as $\Delta t_{i}=t_{i+1}-t_{i}$. This is shown in Fig. 2(b). In the following, $v_{t h}$ has been set to the mean value of $\bar{v}(t)$, denoted as $\langle v\rangle$. Noticeably, $\langle v\rangle=c / k$.

The so-obtained series of avalanches are finally decomposed into $A S$ sequences. Seismologists have developed 

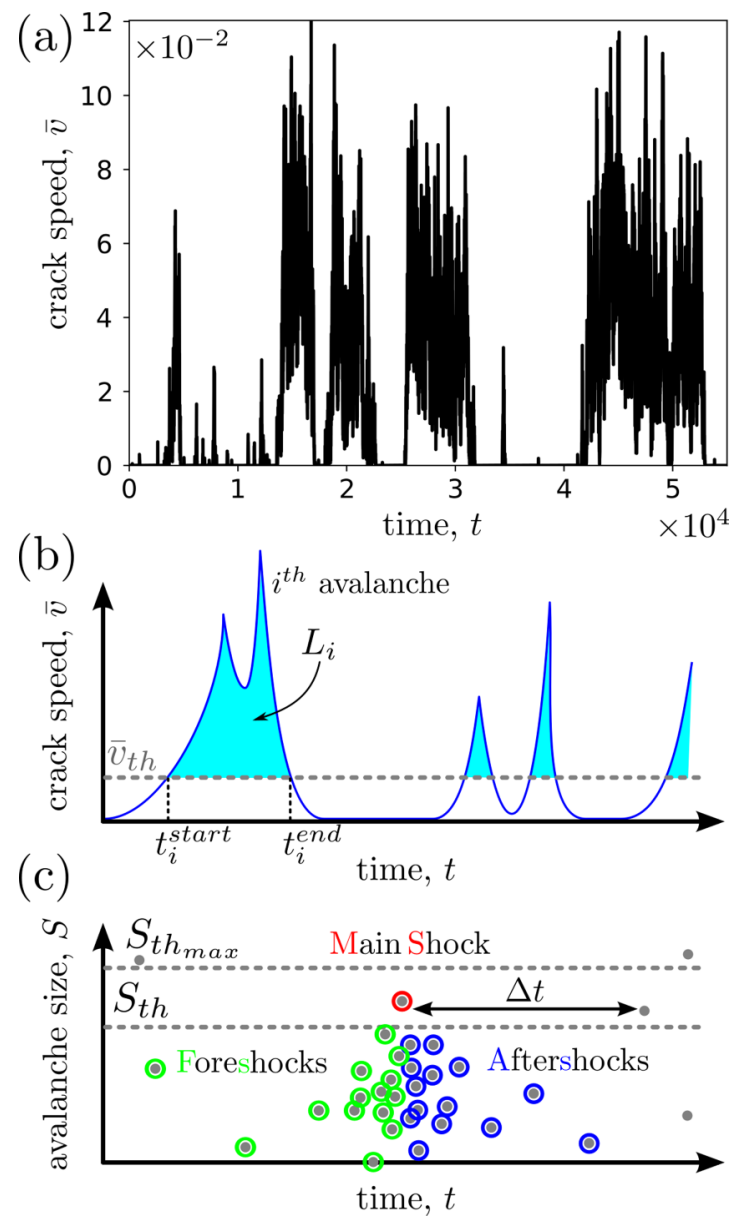

FIG. 2. (a) Example of a mean crack speed signal, $\bar{v}(t)$. Here, $c=2 \times 10^{-6}$ and $k=1 \times 10^{-4}$. Each speed peak corresponds with the crack front jump also called an avalanche. (b) Sketch of a mean crack speed signal. Crack speed peak $i$ larger than a threshold $\bar{v}_{t h}=$ $c / k$ is detected as an avalanche starting at time $t_{i}^{\text {start }}$ and ending at time $t_{i}^{\text {end }}$. The distance swept by the crack front during this avalanche is the area below the peak, $L_{i}$, which gives an avalanche size $S_{i}=$ $N \times L_{i}$. (c) Procedure sketch to identify the $A S$ sequence following a $M S$ (red dot) of size $S_{M S}$ falling within a prescribed range $S_{t h}$ to $S_{\mathrm{th}_{\max }} \gtrsim S_{\mathrm{th}}$. The following events until an event of size larger than $S_{M S}$ is encountered are considered as $A S$ (blue points). Along the same line, the preceding events are considered as $F S$ (green points). The waiting time $\Delta t$ is measured between consecutive events larger than a size threshold $S_{t h}$.

powerful declustering methods in this context (see, e.g., Ref. [78] for a recent review). Most of these methods are based on the spatiotemporal proximity of the events. The spatial proximity is not relevant in this situation with a single crack and hence we adopted the procedure proposed in [8-10,45,55] and sketched in Fig. 2(c) as follows.

(i) All events with energies in a predefined interval between $S_{t h}$ and $S_{t h_{\max }}$ are considered as $M S$.

(ii) The $A S$ sequence associated with each $M S$ is made of all events following this $M S$, till an event of size equal or larger than the $M S$ energy, $S_{M S}$, is encountered.

Foreshocks $(F S)$ are defined the same way after having reversed the time direction.

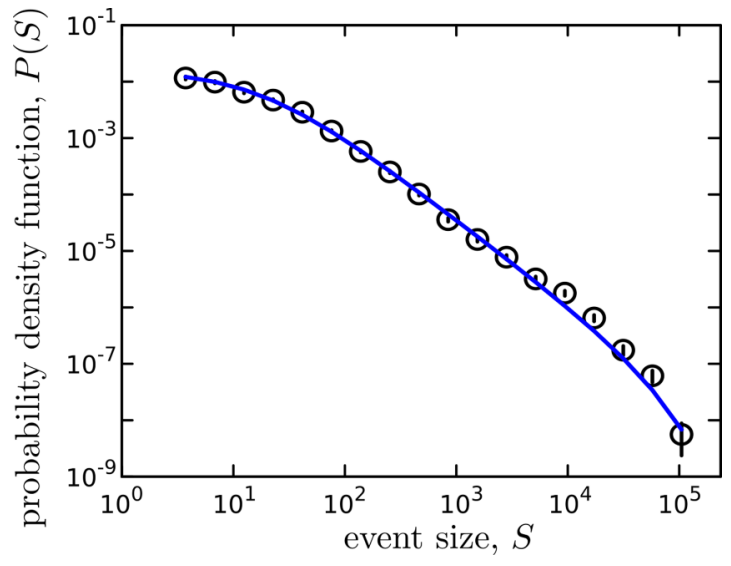

FIG. 3. Probability density function of the event sizes $S$ in a simulation with $c=2 \times 10^{-6}$ and $k=10^{-4}$. The axes are logarithmic. The blue plain curve is a fit by Eq. (11), with exponent $\beta=1.51 \pm$ 0.05 , lower cutoff $S_{\min }=21.3$, and upper cutoff $S_{\max }=1.04 \times 10^{5}$.

\section{SEISMICLIKE ORGANIZATION OF DEPINNING EVENTS}

\section{A. Size distribution and Gutenberg-Richter law}

Figure 3 shows the probability density function (PDF) to observe an event of size $S$ for a typical simulation. The power-law distribution expected for the crackling system is observed over typically four decades. The whole distribution is well fitted by

$$
P(S) \sim \frac{e^{-S / S_{\max }}}{\left(1+S / S_{\min }\right)^{\beta}},
$$

where $S_{\min }$ and $S_{\max }$ are the upper and lower cutoffs of the power-law distribution respectively and $\beta$ is the exponent. Both cutoffs depend on the parameters $c$ and $k$. We will return in Sec. IV $\mathrm{C}$ to the analysis of these dependencies. Conversely, the size exponent, $\beta=1.51 \pm 0.05$, barely depends on these parameters (Fig. 3), as expected near the depinning critical point of a long-range elastic interface within a random potential. Note that the measured exponent is larger than the one expected in the limit of vanishing driving rate: $\beta(c \rightarrow 0) \simeq 1.28$ [2]. As discussed in [57], the measure of an apparent, anomalously large Gutenberg-Richter exponent is the signature of avalanche fragmentation in clusters of smaller avalanches strongly correlated in time.

\section{B. Number of events in $A S$ sequences and productivity law}

We now turn to the $A S$ sequences and test whether the scaling laws of seismicity are fulfilled. Figure 4 presents the mean number of $A S, N_{A S}$, as a function of the size $S_{\text {th }}$ prescribed for the triggering $M S$. In between two cutoffs, $N_{A S}$ goes as a power law with $S_{t h}$ as expected from the productivity law. Following [45], we checked that the $N_{A S}$ vs $S_{M S}$ curve remains unchanged after (i) having reattributed to each event $i$ the energy of another event $j$ chosen randomly and (ii) having arbitrarily set to unity the time interval between two successive events.

This demonstrates that the productivity law is a simple consequence of the size distribution. The relation between 


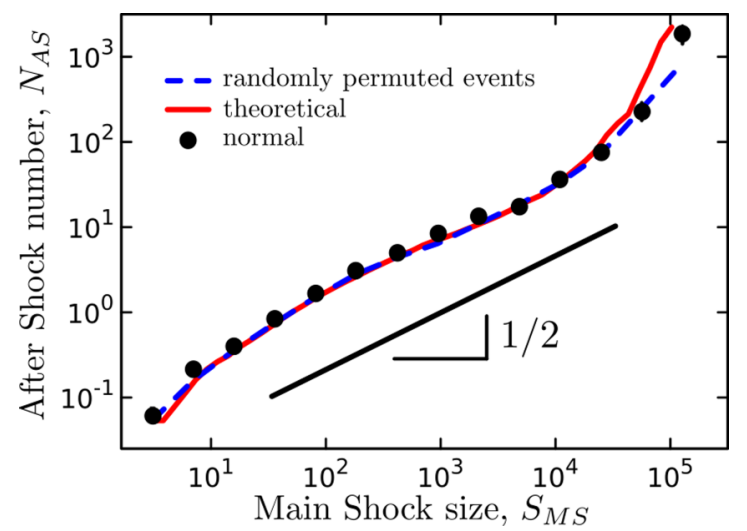

FIG. 4. Mean number of $A S$ in the sequence, $N_{A S}$, as a function of the $M S$ size, $S_{M S}\left(c=2 \times 10^{-6}\right.$ and $\left.k=10^{-4}\right)$. The axes are logarithmic. Black straight line shows an exponent $\alpha=1 / 2$. Black points are the real data and blue dashed line those obtained after having permuted the sizes and set time step to unity. Plain red curve is the solution provided by Eq. (12).

the two can be rationalized using the argument provided in [45,55]: The total number of events with a size larger than the prescribed value $S_{M S}$ gives, by definition, the total number of $M S$ of size $S_{M S}$ and hence the total number of $A S$ sequences. The total number of events with a size smaller that $S_{M S}$ gives the total number to be labeled $A S$ in the catalog. The ratio of the latter to the former gives the mean number of $N_{A S}\left(S_{M S}\right)$. Calling $F(S)$ the cumulative distribution for event size, one gets

$$
N_{A S}\left(S_{M S}\right)=\frac{F\left(S_{M S}\right)}{1-F\left(S_{M S}\right)} .
$$

This equation allows reproducing perfectly the data (plain line in Fig. 4). No fitting parameter is required here. In the scaling regime, $P(S) \sim S^{-\beta}$ with $\beta \approx 1.5$. Hence $F(S) \sim$ $S^{1-\beta}$ and $N_{A S} \sim S_{M S}^{\alpha}$ with $\alpha=\beta-1 \approx 1 / 2$.

\section{Size of the largest aftershock and Båth law}

The next step is to look at the size ratio between a $M S$ and its largest $A S$. Such a curve is presented in Fig. 5. Once again, permuting randomly the events and setting arbitrarily the time step to unity do not modify the curve. As for the productivity law, this means that this law finds its origin in the size distribution only. Following [45], the relation between the two can be derived analytically using extreme value theory (EVT) arguments: let us call $F_{A S_{\max }}\left(S \mid N_{A S}\right)$ the probability that the largest $A S$ of a sequence of size $N_{A S}$ is smaller than $S$. All the other $A S$ in the sequence have a size smaller than $S$ so that $F_{A S_{\max }}\left(S \mid N_{A S}\right)=F(S)^{N_{A S}}$. The mean value $\left\langle\max \left(S_{A S} \mid S_{M S}\right)\right\rangle$ of the size of the largest event over the sequences triggered by a $M S$ of size $S_{M S}$ then writes

$$
\begin{aligned}
\left\langle\frac{\max \left(S_{A S}\right)}{S_{M S}}\right\rangle= & N_{A S}\left(S_{M S}\right) \\
& \times \int_{S_{\min }}^{S_{M S}} S F(S)^{N_{A S}\left(S_{M S}\right)-1} P(S) d S,
\end{aligned}
$$

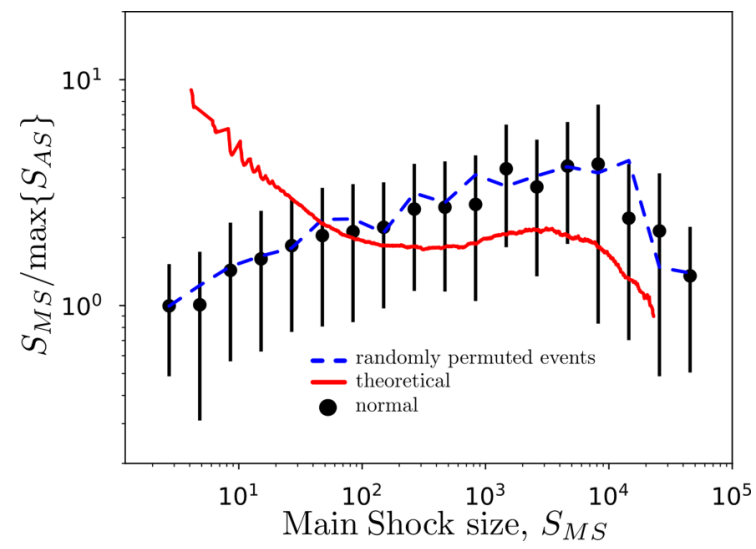

FIG. 5. Mean size ratio $\max \left(S_{M S}\right) / S_{A S}$ between a $M S$ and its largest $A S$, plotted as a function of the $M S$ size, $S_{M S}\left(c=2 \times 10^{-6}\right.$ and not as $k=10^{-4}$ ). The axes are logarithmic. Black points are the real data and blue dashed line those obtained after having permuted the sizes and set time step to unity. Plain red curve is the solution provided by Eq. (13).

where $N_{A S}\left(S_{M S}\right)$ is given by Eq. (12). This analytical solution gives a fairly good prediction of the order of $\max \left(S_{A S}\right) / S_{M S}$ (see Fig. 5) provided the fact that there is no fitting parameter. A discrepancy is however observed at low $S_{M S}$. This is because the number of $A S$ produced by each $M S$ is small there and hence the EVT argument provided to derive Eq. (13) is not relevant anymore.

\section{Distribution of interevent time and Bak et al. law}

We now turn to the analysis of the occurrence time of avalanches. Scale-free statistics is observed for the waiting time separating two successive avalanches; as for avalanche sizes, the whole distribution is well fitted by [Fig. 6(a)]:

$$
P(\Delta t) \sim \frac{e^{-\Delta t / \Delta t_{\max }}}{\left(1+\Delta t / \Delta t_{\min }\right)^{\gamma}},
$$

where the two time cutoffs $\Delta t_{\min }$ and $\Delta t_{\max }$ bound the scale free statistics and $\gamma$ refers to the exponent in between. Same statistics is observed when only the events of size larger than a prescribed threshold, $S_{t h}$, are considered [Fig. 6(a)]. The parameters $\gamma$ and $\Delta t_{\max }$ barely depend on $S_{t h}$. Conversely, the lower cutoff $\Delta_{\min }$ increases with $S_{t h}$. As observed for seismic events $[27,28]$ or for $\mathrm{AE}$ produced in fracture experiments at laboratory scale $[8-10,45,55,79]$ and for sheared granular material [54], all curves collapse onto a single master curve [Fig. 6(c)], once time is rescaled with the activity rate $R\left(S_{t h}\right)$, defined as the total number of events divided by the simulation duration:

$$
P\left(\Delta t \mid E_{t h}\right) \sim R\left(S_{t h}\right) f\left[u=R\left(S_{t h}\right) \Delta t\right],
$$

with $f(u) \sim(1+u / b)^{-\gamma} e^{-u / B}$. The fact that $f(u)$ takes the form of a gamma distribution underpins a stationary statistics for the event series $[10,28,45]$. The two rescaled time cutoff $b$ and $B$ relates to $\Delta t_{\min }$ and $\Delta t_{\max }$ via $b=R \Delta t_{\min }$ and $B=$ $R \Delta t_{\max }$, where $R$ denotes the mean activity rate during the simulation (total number of avalanches divided by the total duration of the simulation). These three parameters $\gamma, b$, and $B$ can be interrelated using the conditions $\int_{0}^{\infty} f(u) d u=1$ 

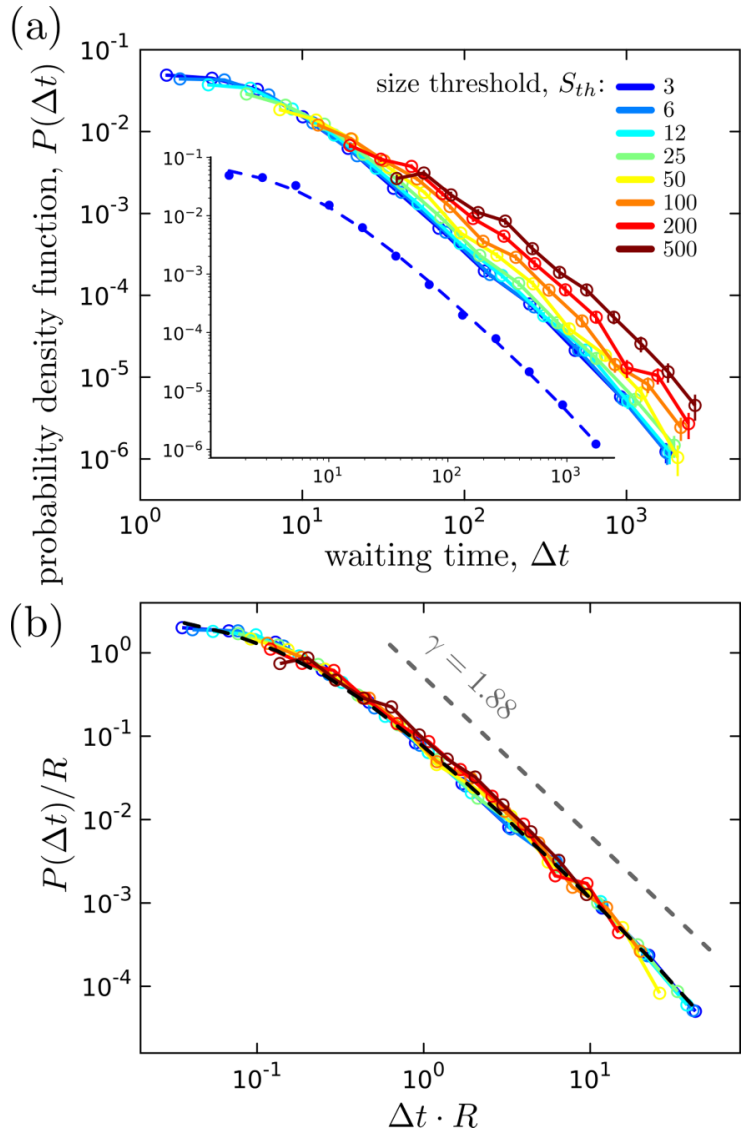

FIG. 6. (a) Probability density functions of the waiting time $\Delta t$ between two consecutive events of size larger than a prescribed threshold $S_{t h}$. Here, $c=1 \times 10^{-5}$ and $k=5 \times 10^{-4}$. The different curves correspond to different values $S_{t h}$. In the inset the dots are the curve reproduced for $S_{t h}=3$ and the plain curve is a fit by Eq. (14), with exponent $\gamma=1.88 \pm 0.09$, lower cutoff $\Delta t_{\min }=6.0$, and upper cutoff $\Delta t_{\max }=3.8 \times 10^{3}$. (b) Collapse obtained after having rescaled $\Delta t$ with the mean activity rate $R\left(S_{t h}\right)$. Straight dashed line is a power law of fitted exponent $\gamma=1.88$. Black dashed curve is the rescaled fitted curve of the curve in the inset of panel (a). In both panels (a) and (b), the axes are logarithmic. Vertical bars stand for $95 \%$ error bars.

[normalization of the probability density function $P\left(\Delta t \mid E_{t h}\right)$ ] and $\int_{0}^{\infty} u f(u) d u=1\left[\right.$ since $\left.\langle\Delta t\rangle=1 / R\left(S_{t h}\right)\right]$.

\section{E. Production rate of $A S$ and Omori-Utsu law}

Finally, we looked at the rate of $A S$ produced by a $M S$ of size $S_{M S}$ and its evolution as a function of the time elapsed since $M S: R_{A S}\left(t-t_{M S} \mid E_{M S}\right)$. To compute these curves, we adopted the procedure developed in [45]: for each simulation, all sequences triggered by $M S$ of size falling within a prescribed interval are sorted out; subsequently the $A S$ events are binned over $t-t_{M S}$ and the so-obtained curves are finally averaged. Figure 7 shows the resulting curves in a typical simulation. An algebraic decay compatible with the OmoriUtsu law [50,52] is observed [see Fig. 7(a)] and, within the error bar, the Omori exponent is equal to the exponent $\gamma$
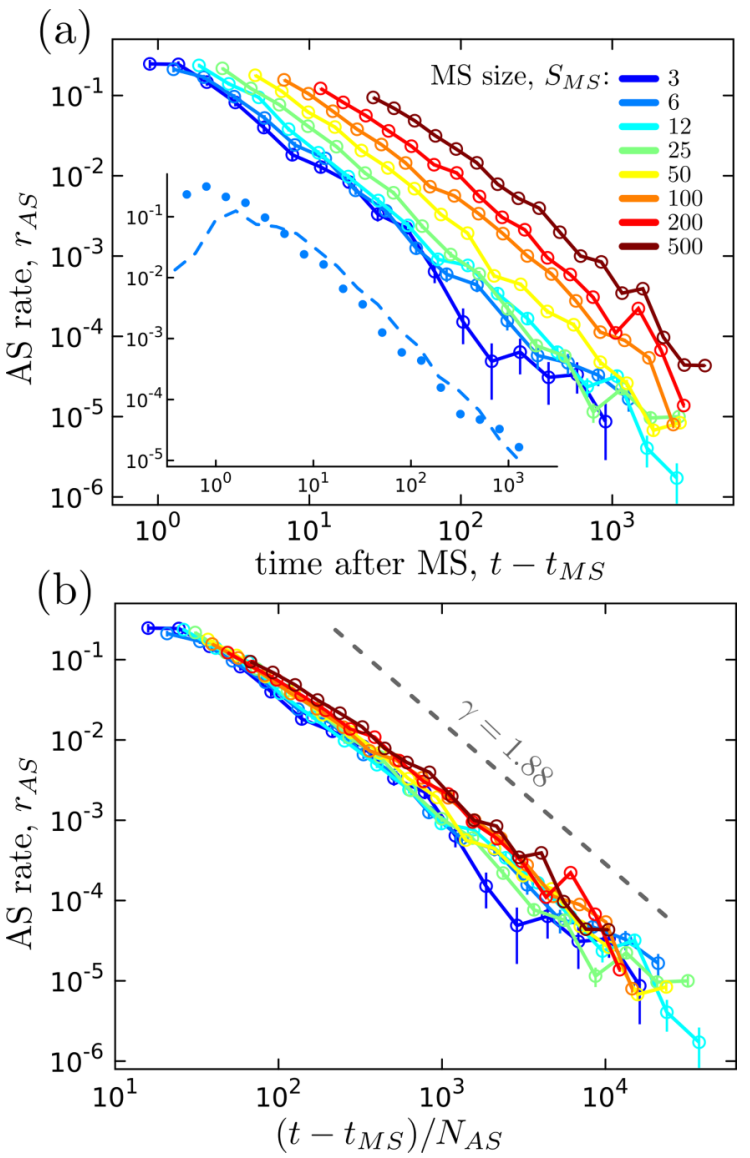

FIG. 7. (a) Rate of AS, $R_{A S}\left(t-t_{M S} \mid S_{M S}\right)$, triggered by a $M S$ of size $S_{M S}$ plotted as a function of the time elapsed since $M S$, $t-t_{M S}$. Here, $c=1 \times 10^{-5}$ and $k=5 \times 10^{-4}$. The different curves correspond to different values of $S_{M S}$. In the inset the dots are the curve reproduced for $S_{t h}=6$ and the dashed curve is obtained after having permuted randomly the size $S_{i}$ attributed to each event occurring at $t_{i}$. (b) Collapse obtained after having set $t-t_{M S} \rightarrow(t-$ $\left.t_{M S}\right) / N_{A S}\left(S_{M S}\right)$, where $N_{A S}\left(S_{M S}\right)$ is the mean number of $A S$ produced by a $M S$ of size $S_{M S}$ and is given by Eq. (12). Straight dashed line is a power law of exponent $\gamma=1.88 \pm 0.09$ obtained from the analysis of interevent time [see Fig. 6 and Eq. (14)].

associated with $P(\Delta t)$ :

$$
R_{A S}(t) \sim \frac{1}{\left(t-t_{M S}\right)^{\gamma}} .
$$

As in [45], permuting randomly the event sizes in the initial series does not modify the curves observed in Fig. 7. Hence Omori-Utsu law and the time dependency of $R_{A S}\left(t \mid S_{M S}\right)$ find their origin in the scale-free distribution of $P(\Delta t)$ and hence the Omori-Utsu exponent is equal to $\gamma$ [45] and is found to be independent of the $M S$ size $S_{M S}$. Finally, following [45], we checked that the dependency with $S_{M S}$ can be fully captured by rescaling $t-t_{M S} \rightarrow\left(t-t_{M S}\right) / N_{A S}\left(S_{M S}\right)$ [see Fig. 7(b)].

As in [45], all curves collapse onto a master curve once $t-$ $t_{M S}$ is rescaled by the mean number of $A S, N_{A S}\left(S_{M S}\right)$, produced by a $M S$ of size $S_{M S}$ :

$$
R_{A S}\left(t \mid S_{M S}\right) \sim \frac{1}{\left(1+\frac{t-t_{M S}}{\tau_{\min } N_{A S}}\right)^{\gamma}} .
$$


The very same relation holds for the $F S$ rate $R_{F S}\left(t_{M S}-t\right)$ as the event series are stationary [45].

\section{EFFECT OF LOADING SPEED AND UNLOADING RATE}

\section{A. On the selection of size distribution}

We now turn to the role played by the control parameters, namely the (dimensionless) driving rate $c$ and unloading factor $k$ in Eq. (9), onto the dynamics exhibited by the crack front. Figures 8(a) and 8(b) present the size distribution $P(S)$ obtained at different $k$ and $c$. Four observations emerge.

(i) The lower cutoff $S_{\min }$ increases with increasing $c$ and decreasing $k$.

(ii) At fixed $c$, the upper cutoff $S_{\max }$ displays a nonmonotonic behavior with $k$. It first increases with $k$ at small $k$, reaches a maximum at $k^{*}$, and decreases at larger $k$. The increasing phase and the maximum position $k_{*}$ depend on $c$. Conversely, the decreasing phase seems independent of $c$.

(iii) Over the whole range explored, $P(S)$ is in first approximation compatible with the gamma distribution (with lower cutoff) provided by Eq. (11).

(iv) The exponent $\beta$ (slope in the log-log representation) barely depends on $c$.

The lower and upper cutoffs of $P(S)$ are either measured directly by fitting the experimental curves with Eq. (11) or by using

$$
S_{\min }=1 /\langle 1 / S\rangle, \quad S_{\max }=\left\langle S^{2}\right\rangle /\langle S\rangle .
$$

It was checked that both definitions lead to the same results, but for a prefactor close to unit.

The lower cutoff is found to increase almost linearly with $\langle v\rangle=c / k$ [see Fig. 8(c)]:

$$
S_{\min }(c, k) \sim\langle v\rangle .
$$

The saturation of $P(S)$ for $S \leqslant S_{\min }$ may also be a consequence of the prescribed threshold $v_{t h}=\langle v\rangle$. Indeed, by setting a small and constant threshold $v_{t h}$, it has been shown [67] that neither $c$ nor $k$ affect the value of $S_{\min }$.

The upper cutoff, $S_{\max }$, displays a nonmonotonic behavior with $k$. This behavior can be qualitatively understood in the framework of the depinning transition. At small velocity $\langle v\rangle$, the quasistatic limit is reached and each burst corresponds to a single depinning avalanche. In this limit, the avalanche statistics is scale-free up to a correlation length $\xi_{k} \sim 1 / \sqrt{k}$ [2]. When $\langle v\rangle$ increases, a second velocity dependent length scale is involved:

$$
\xi_{v} \sim\langle v\rangle^{-v / \theta},
$$

with $v=1.625$ and $\theta=0.625[2,80]$. The cutoff $S_{\max }$ is governed by this length scale when $\xi_{v} \leqslant \xi_{k}$. The crossover between these two regimes occurs when $\xi_{v} \sim \xi_{k}$, that is

$$
k^{*} \sim c^{2 v /(\theta+2 v)} .
$$

In the framework of the depinning transition, $S_{\max }$ is then expected to evolve with $c$ and $k$ as

$$
\begin{gathered}
S_{\max }(c, k) \sim k^{-(1+\zeta) / 2} g\left(u=c / k^{1+\theta / 2 v}\right), \\
\text { with } g(u) \sim \begin{cases}1 & \text { if } u \ll 1, \\
u^{-v(1+\zeta) / \theta} & \text { if } u \gg 1,\end{cases}
\end{gathered}
$$
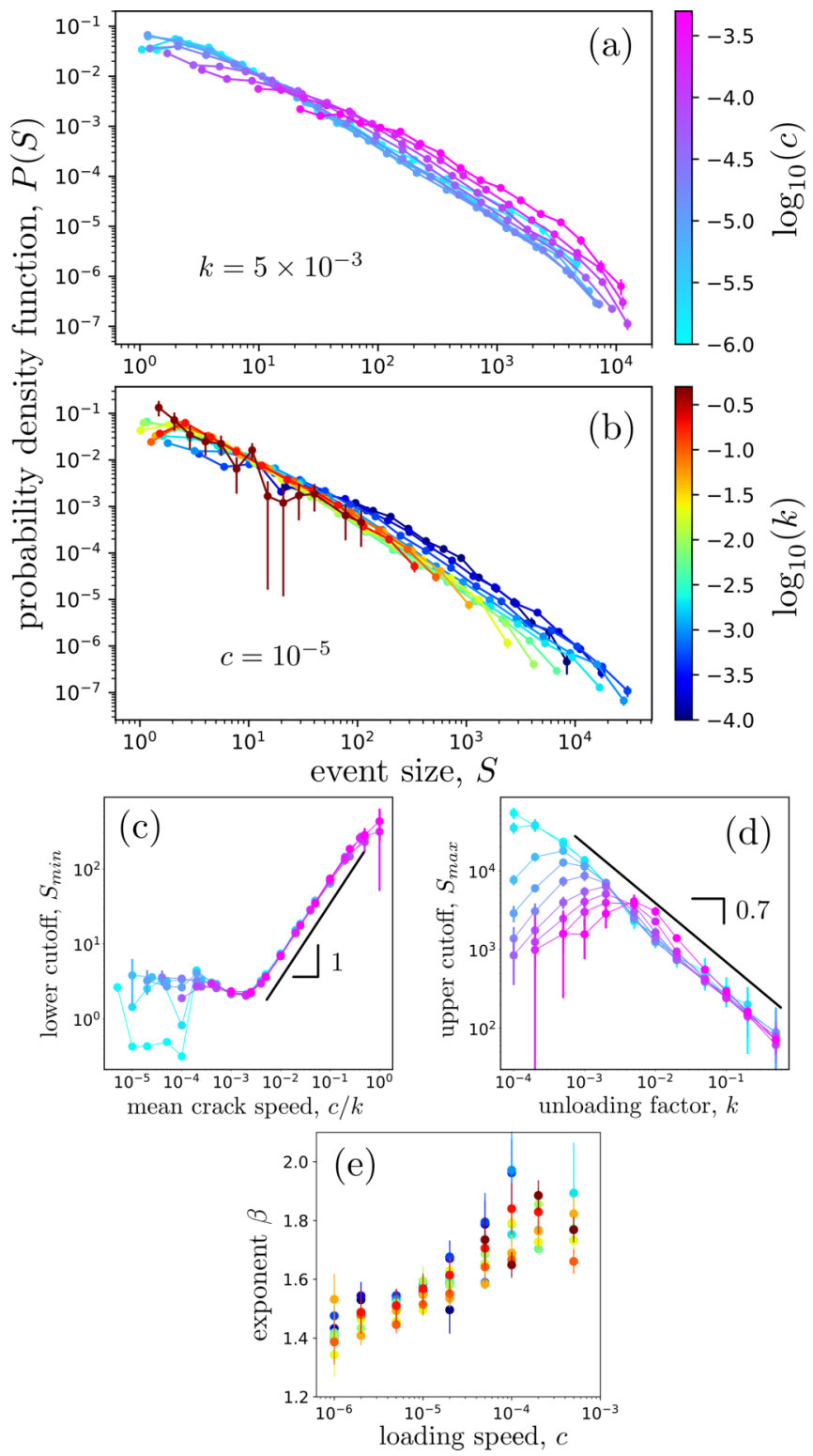

FIG. 8. Probability density function of the avalanche size $P(S)$ for different loading speed $c \in\left[10^{-6}, 5 \times 10^{-4}\right]$ keeping $k=5 \times$ $10^{-3}$ (a) and for different unloading factors $k \in\left[10^{-4}, 5 \times 10^{-1}\right]$ keeping $c=10^{-5}$ (b). $P(S)$ follows a power law with exponent $\beta$ in between two cutoffs, $S_{\min }$ and $S_{\max }$. These three parameters are then determined by fitting each $P(S)$ curve using Eqs. (11) and (18). (c) Evolution of the so-obtained lower cutoff $S_{\min }=\frac{1}{\langle 1 / S\rangle}$ as a function of $\langle v\rangle$. The dependency is almost linear; the black straight line has a slope of 1. (d) Evolution of the upper cutoff $S_{\max }=\frac{\left\langle S^{2}\right\rangle}{\langle S\rangle}$ as a function of $k$ for different values of $c . S_{\max }$ decays as a power law with $k$, with a fitted exponent $\sim 0.7$ (black straight line). (e) Exponent $\beta$ as a function of $c$ for different $k$ values. $\beta$ (very) slightly decreases with increasing $c$. On average, it remains close to $\sim 1.5$. On all panels, error bars stand for $95 \%$ confident interval.

where the roughness exponent $\zeta=0.4$ [24]. Note that this prediction holds in the continuum limit, when finite size and discretization effect can be neglected: $1 \ll\left\{\xi_{k}, \xi_{v}\right\} \ll L$. In Fig. 8(d), we show the nonmonotonic behavior of $S_{\max }$ with $k$ and the agreement between the data and Eq. (22) 


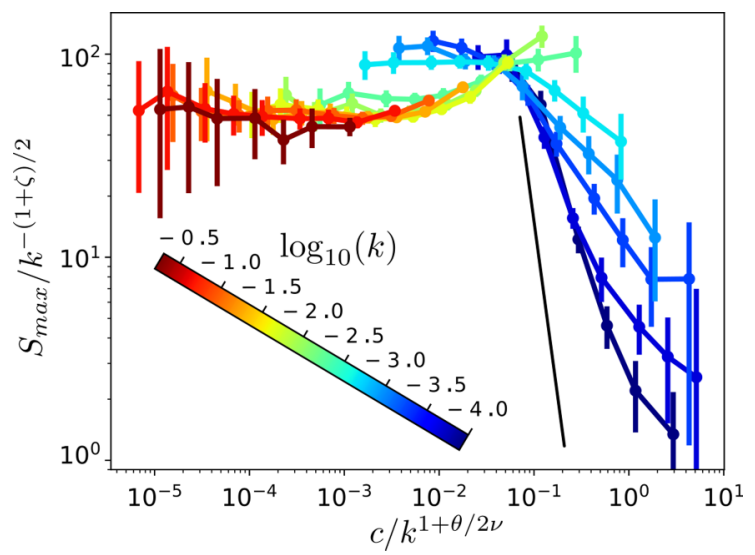

FIG. 9. Evolution of $S_{\max } / k^{-(1+\zeta) / 2}$ vs $c / k^{1+\theta / 2 v}$. A collapse of all curves is predicted within the depinning transition framework [Eq. (22)]. This collapse is fulfilled for large $k$ and small $c$. Conversely, it is not fulfilled at large $c$ and/or small $c$. This departure results from size and discretization effects. Straight black line indicates the power law of exponent $-v(1+\zeta) / \theta \simeq-3.64$ predicted within the depinning transition framework.

for large $k$. To go deeper into the comparison, we looked at the variation of $S_{\max }$ as a function of $c$ at fixed $k$. Figure 9 shows $S_{\max } / k^{(1+\zeta) / 2}$ vs $c / c^{*}$ with $c^{*} \sim k^{1+\theta / 2 v}$. For small $c$ we found the collapse of the plateau consistent with the large scale $k$ behavior of Eq. (22). For larger values of $c, S_{\max }$ decreases with increasing $c$ as $\xi_{v}$ is dominant. The power law predicted by Eq. (22) is shown by the plain black line and the agreement is not fulfilled. This departure results from size and discretization effects: at large $k, \xi_{v}$ starts being dominant only at short length scales. At smaller $k, \xi_{v}$ is larger and the decay approaches the expected one but the system size is too small as can be seen from the noncollapse of the plateau.

The distribution $P(S)$ is well fitted here by the gamma distribution provided in Eq. (11). It is worth noting that, in the quasistatic limit $\left(\langle v\rangle \rightarrow 0\right.$ and subsequently $\left.v_{t h} \rightarrow 0\right), P(S)$ displays a stretched exponential behavior with exponents that can be computed by FRG techniques [40].

Within error bars, $\beta$ is independent of $k$. Conversely, it increases slightly with $c$, from $\sim 1.4$ at $c=10^{-6}$ to $\sim 1.8$ at $c=10^{-4}$ [see Fig. 8(e)]. The value at vanishing $c$ is in agreement with the FRG value $\beta(c \rightarrow 0)=1.28$ [2]. The larger value observed at finite $c$ may be an effect of the finite threshold, which, by dividing the depinning avalanches into smaller ones, could yield a larger effective exponent $\beta$ [57]. Indeed, similar to what has already been discussed for $S_{\min }$, making a different choice for the prescribed threshold $v_{t h}$, that is, setting it to a constant prescribed low value $\left(v_{t h}=10^{-3}\right.$ as in [67]) yields a constant $\beta$ contrary to what is observed here. This emphasizes the importance of finite thresholding in the analysis of the selection of scales in crackling dynamics.

\section{B. On the selection of waiting time law}

Figure 10 synthesizes the effect of the parameters $c$ and $k$ onto the distribution of waiting time. The main effect observed
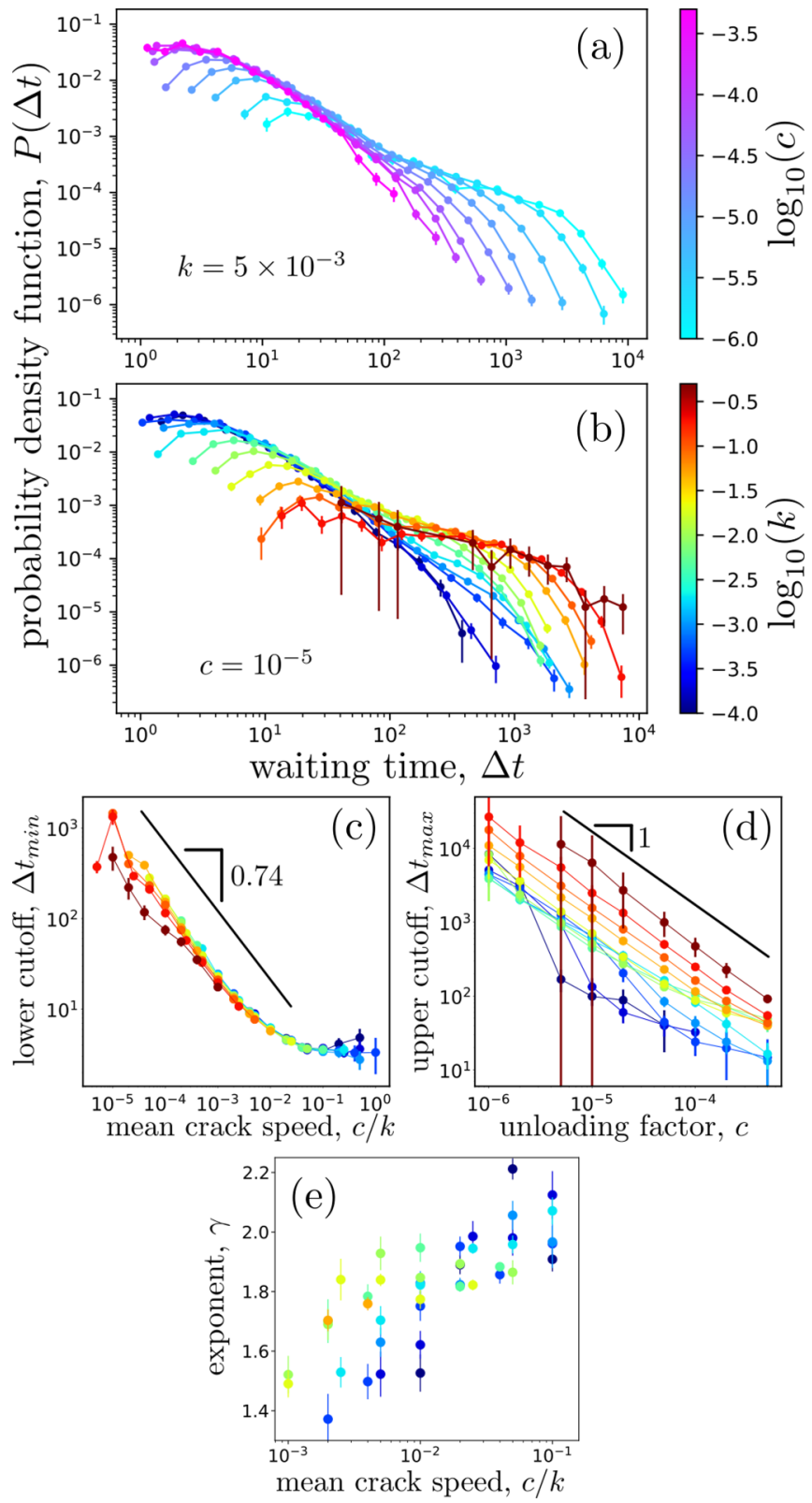

FIG. 10. (a) Probability density function of the waiting time between two consecutive avalanches of size larger than $S_{t h}=4, P(\Delta t)$. Curves are plotted for different loading speed $c \in\left[10^{-6}, 5 \times 10^{-4}\right]$ keeping $k=5 \times 10^{-3}$ (a) and for different unloading factors $k \in$ $\left[10^{-4}, 5 \times 10^{-1}\right]$ keeping $c=10^{-5}$ (b). $P(\Delta t)$ follows a power law with exponent $\gamma$ in between two cutoffs $\Delta t_{\min }$ and $\Delta t_{\max }$. These three parameters are then determined by fitting each $P(\Delta t)$ curve using Eq. (14) and equations equivalent to Eq. (18) for $\Delta t$. (c) Evolution of $\Delta t_{\text {min }}$ as a function of the mean crack speed $\langle v\rangle=c / k . \Delta t_{\text {min }}$ decays as a power law with $\langle v\rangle$, with a fitted exponent close to 0.74 (black straight line). (d) Evolution of $\Delta t_{\max }$ as a function of c. $\Delta t_{\max }$ decays as a power law with $c$, with a fitted exponent close to 1 (black straight line). (e) Exponent $\gamma$ as a function of $\bar{v} \cdot \gamma$ increases logarithmically with $\bar{v}$ and goes from 1.4 at $\bar{v} \simeq$ $10^{-3}$ to 2.2 at $\bar{v} \simeq 10^{-1}$. The different colors in panels (c) to (e) correspond to different values $k$ according to the color bar provided in panel (e). In all panels, the vertical bars stand for $95 \%$ error bars. 
here is that decreasing $c$ and/or increasing $k$ flatten the curve (in logarithmic axis), making the effective exponent $\gamma$ larger [see Figs. 10(a) and 10(b)]; here again, $\langle v\rangle=c / k$, seems to be the relevant parameter and $\gamma$ goes from $\sim 1.4$ to $\sim 2.2$ as $\bar{v}$ goes from $10^{-3}$ to $10^{-1}$ [see Fig. 10(e)]. The value at vanishing speed is close to 1.5 , which corresponds to the exponent of the power-law statistics of the avalanche duration in the quasistatic limit $(\alpha=1+\zeta / \kappa \simeq 1.50$, where $\kappa=0.77$ is the dynamic exponent for the long range depinning transition [80]). This scaling symmetry between the waiting time statistics and the avalanche duration statistics has indeed been invoked in [63] when a finite threshold $v_{t h}=\langle v\rangle$ is prescribed. The increase of $\gamma$ with $\bar{v}$ is similar to what is observed experimentally in [45].

In contrast to what has been observed for the size $S$ (Sec. IV A), both the minimal and maximal waiting times $\Delta t_{\min }$ and $\Delta t_{\max }$ decreases with $\bar{c}$ (or $\langle v\rangle$ ) [Figs. 10(c) and 10(d)]. This can be understood if one thinks that the nucleation rate of new avalanches is proportional to $c$. Hence the typical waiting time, $\widetilde{\Delta t}$, between successive avalanches goes as $1 / c$. Indeed, as long as the duration of the avalanche is negligible, in order to nucleate a new avalanche, one should increase the force $\delta F=c \delta t$ by a fixed amount $\sim 1 / L$ [81].

This scaling is perfectly obeyed by $\Delta t_{\max }$ for large $k$ and small $c$. When $k$ decreases, avalanche duration becomes larger. This induces a decrease of the measured $\Delta t_{\max }$, which does not coincide exactly with the time interval between successive nucleation events anymore. In this regime, the $1 / c$ scaling is only an upper bound for $\Delta t_{\max }$ that is shifted all the more so as $k$ decreases. This regimes survives as long as the avalanche duration remains small with respect to $\widetilde{\Delta t}$. As the upper cutoff is mainly limited by $\xi_{k}$ (see Sec. IV A), this avalanche duration is expected to increase with decreasing $k$ and, for small enough $k$, to become of the order of $\widetilde{\Delta t}$. At this point, the depinning avalanches coalesce together and the waiting time in between drops abruptly. In this coalescence regime, it is the finite threshold value $(c / k)$ that controls $\Delta t_{\max }$.

\section{On the conditions leading to seismiclike organization}

Finally, to unravel the conditions favoring seismiclike behavior, that is, a scale free statistics of size and waiting time, we plotted, in Fig. 11, the number of decades over which a scale free statistics is observed for both quantities.

Concerning the sizes, two zones with many decades of scale-free statistics are observed [Fig. 11(a)]: a first, fairly large, one in the left-handed-lower part of the diagram (small $k$, small $c$ ) and a second smaller one at the left-handed-upper part (finite $k$, small $c$ ). The fact that $c$ should be small is well understood: small $c$ yields small $\langle v\rangle$, which favors both large $S_{\max }$ and small $S_{\min }$ [see Figs. 8(c) and 8(d)]. Conversely, $k$ has two antagonist effects: increasing $k$ makes $\xi_{k}$ small, hence preventing large $S_{\max }$; but, at the same time, it makes $\langle v\rangle$ small, yielding small $S_{\min }$. The existence of the small zone with scale-free statistics at moderate $k$ and small $c$ is a consequence of this small $S_{\min }$; it cannot be understood within the depinning theory but is a direct consequence of the experimental choice of a finite threshold equal to $\langle v\rangle$.
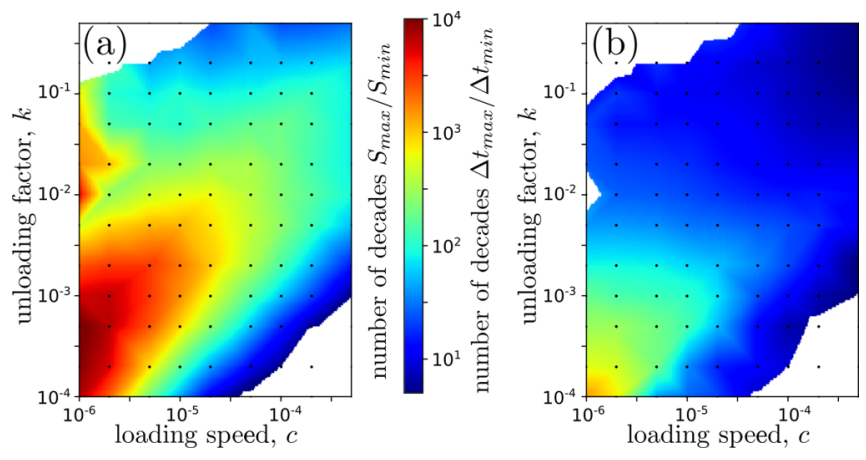

FIG. 11. Phase diagram showing the $\{c, k\}$ conditions to observe: (a) crackling dynamics, that is scale-free statistics for size over a significant number of decades, and (b) temporal seismiclike intermittency, that is a scale-free statistics for interevent time over a significant number of decades. In both maps, the $c$ and $k$ axes are logarithmic. The color indicates $\log _{10}\left(S_{\max } / S_{\min }\right)$ (a) and $\log _{10}\left(\Delta t_{\max } / \Delta t_{\min }\right)$ (b) according to the color bar shown in between the two panels. The range of parameters $\{c, k\}$ allowing one to observe extended scale-free statistics for size is much larger than that required to observe extended scale-free temporal correlation [red areas in (a) and (b)]. The white spaces in the high $c$-low $k$ and high $k$-low $c$ regions of both panels (a) and (b) coincide to regions where the number of decades over which the power law is observed is too small to be properly determined.

Concerning the time clustering at the origin of the dynamics intermittency and of the fundamental seismic laws (see Sec. III), the scale free statistics is observed only in a tiny region with both small $k$ and $c$. Small $c$ is needed to observe large $S_{\max }$ [Fig. 10(d)] and small $k$ is needed to get large $\langle v\rangle$, and subsequently small $S_{\min }$. Note that the extension of the $\{c, k\}$ domain which allows observing scale-free interevent times over a significant range of scale is much smaller than that required for observing scale-free sizes. This explains why time clustering and seismiclike organization of avalanche sequences are barely reported in the context of depinning interfaces.

\section{CONCLUDING DISCUSSION}

We analyzed here crackling dynamics exhibited by a longrange elastic $1 \mathrm{D}$ interface driven in a random potential. A slow and constant loading rate, $c$, is imposed and a finite unloading factor, $k$, is considered. As a result, the force applying onto the interface self-adjusts around the depinning threshold and the motion exhibits a steady avalanche dynamics, with a speed signal $v(t)$ fluctuating highly around an average value $\langle v\rangle=$ $c / k$. The avalanches were identified with the bursts above this mean value, and their size and occurrence time were collected in event catalogs.

The analysis of these catalogs revealed a statistical organization similar to that reported in sismology: both the avalanche size and interevent time are power-law distributed. Moreover, the events form aftershock sequences obeying the fundamental laws of seismology: productivity law with a mean number of produced aftershocks scaling as a power law with the main shock size, Båth's law with a ratio between the size of the main shock and that of its largest aftershock is 
constant, and Omori-Utsu law with an aftershock productivity rate decaying as a power law with time. As experimentally observed in [45], these laws do not reflect some nontrivial correlation between size and occurrence time: they directly emerge from the scale-free statistics of energy (for the productivity and Båth's laws) and from that of interevent time (for Omori's laws).

The value of the loading rate and unloading factor has a drastic effect on the scaling exponents associated with the scale-free statistics of size and interevent time on one hand and on the lower and upper cutoff limiting the scale-free regime on the other hand. The framework of the depinning transition allows for understanding some of this effect-the dependency of $S_{\max }$ with $k$ and that of $\Delta t_{\max }$ with $c$ in particular. Still, this framework presupposes a quasistatic dynamics $(c \rightarrow 0)$. A finite driving rate, e.g., requires us to work with a finite thresholding, which is shown here to have a drastic effect on the selection of $S_{\min }$ and $\Delta t_{\min }$. This finite thresholding has also been invoked to be responsible for the scale-free statistics of interevent times [44,63]. By making the depinning avalanches overlap partially, a finite driving rate also affects the effective values of the scaling exponents for size and interevent time [79]. Note finally that the dependencies of the lower and upper cutoffs with loading rate and unloading factor make it nontrivial to predict when crackling (scale-free size statistics) and/or seismiclike (scale-free statistics for both size and interevent time) are observed. Small values for both $c$ and $k$ are required for the latter, while small $c$ and even moderate $k$ permits one to observe crackling. This is found to be in qualitative agreement with fracture experiment in model rocks $[45,82]$ : when the loading speed is increased, crackling dynamics and time clustering are not observed anymore.

Crackling dynamics is quite generic in nature and the paradigm of the depinning transition applies to a variety of physical, biological, and social systems. Beyond the crack problems, the long-range interface model analyzed here is also known to describe other systems, including the dynamics of contact lines in wetting problems [23] and the dynamics of domain walls in ferromagnets [83]. In the second case, the field sweep rate and the demagnetization factor play the same role as $c$ and $k$. The new insights obtained here on the time-size organization of fracture events and its evolution with $c$ and $k$ can be directly transposed to these systems. Qualitatively similar features are also expected in other problems mapping onto the problem of driven elastic interfaces in random media, as e.g., fluid invasion of porous and fractured media $[84,85]$, dislocation dynamics in random alloy [86], or combustion fronts in paper [87]. However, due to the short-range nature of the elastic interactions, the universality class of the exponents will be different.

In the single crack description examined here, the events can form $A S$ sequences obeying the fundamental laws of seismology, as in the more complex multicracking situations of quasibrittle compressive fracture. As such, our results may be instrumental to discuss some of the observations on the Portevin-Le Chatelier effect, which emerge from the collective dynamics of interacting dislocation in random alloys. Those include the effect of the applied strain rate onto the exponent characterizing the scale-free statistics in energy of emitted acoustic pulses $[88,89]$ and the effect of thresholding onto the statistics of waiting time between these acoustic pulses [44].

\section{ACKNOWLEDGMENT}

Support through the ANR project MEPHYSTAR is gratefully acknowledged.
[1] K. J. Måløy, S. Santucci, J. Schmittbuhl, and R. Toussaint, Phys. Rev. Lett. 96, 045501 (2006).

[2] D. Bonamy, J. Phys. D 42, 214014 (2009).

[3] D. Bonamy and E. Bouchaud, Phys. Rep. 498, 1 (2011).

[4] J. Barés, M. L. Hattali, D. Dalmas, and D. Bonamy, Phys. Rev. Lett. 113, 264301 (2014).

[5] A. Petri, G. Paparo, A. Vespignani, A. Alippi, and M. Costantini, Phys. Rev. Lett. 73, 3423 (1994).

[6] S. Zapperi, A. Vespignani, and H. E. Stanley, Nature (London) 388, 658 (1997).

[7] M. J. Alava, P. K. V. V. Nukala, and S. Zapperi, Adv. Phys. 55, 349 (2006).

[8] J. Baro, A. Corral, X. Illa, A. Planes, E. K. H. Salje, W. Schranz, D. E. Soto-Parra, and E. Vives, Phys. Rev. Lett. 110, 088702 (2013).

[9] T. Mäkinen, A. Miksic, M. Ovaska, and M. J. Alava, Phys. Rev. Lett. 115, 055501 (2015).

[10] H. V. Ribeiro, L. S. Costa, L. G. A. Alves, P. A. Santoro, S. Picoli, E. K. Lenzi, and R. S. Mendes, Phys. Rev. Lett. 115, 025503 (2015).

[11] L. P. Kubin and Y. Estrin, J. Phys. III 1, 929 (1991).

[12] M. A. Lebyodkin, Y. Brechet, Y. Estrin, and L. P. Kubin, Phys. Rev. Lett. 74, 4758 (1995).
[13] F. B. Klose, F. Hagemann, P. Hähner, and H. Neuhäuser, Mater. Sci. Eng., A 387-389, 93 (2004).

[14] M. C. Miguel, A. Vespignani, S. Zapperi, J. Weiss, and J. R. Grasso, Nature (London) 410, 667 (2001).

[15] S. Papanikolaou, D. M. Dimiduk, W. Choi, J. P. Sethna, M. D. Uchic, C. F. Woodward, and S. Zapperi, Nature (London) 490 517 (2012).

[16] M. Zaiser, Adv. Phys. 55, 185 (2006).

[17] G. Ananthakrishna, Phys. Rep. 440, 113 (2007).

[18] A. Nicolas, E. E. Ferrero, K. Martens, and J.-L. Barrat, Rev. Mod. Phys. 90, 045006 (2018).

[19] J. Barés, D. Wang, D. Wang, T. Bertrand, C. S. O'Hern, and R. P. Behringer, Phys. Rev. E 96, 052902 (2017).

[20] J. S. Urbach, R. C. Madison, and J. T. Markert, Phys. Rev. Lett. 75, 276 (1995).

[21] G. Durin and S. Zapperi, in The Science of Hysteresis, edited by G. Bertotto and I. Mayergoyz (Academic, New York, 2005), p. 181.

[22] P. L. Doussal, M. Müller, and K. J. Wiese, Europhys. Lett. 91, 57004 (2010).

[23] D. Ertaş and M. Kardar, Phys. Rev. E 49, R2532 (1994).

[24] A. Rosso and W. Krauth, Phys. Rev. E 65, 025101(R) (2002). 
[25] R. Planet, S. Santucci, and J. Ortín, Phys. Rev. Lett. 102, 094502 (2009).

[26] J. H. Snoeijer and B. Andreotti, Annu. Rev. Fluid Mech. 45, 269 (2013).

[27] P. Bak, K. Christensen, L. Danon, and T. Scanlon, Phys. Rev. Lett. 88, 178501 (2002).

[28] A. Corral, Phys. Rev. Lett. 92, 108501 (2004).

[29] C. Langenbruch, C. Dinske, and S. A. Shapiro, Geophys. Res. Lett. 38, L21302 (2011).

[30] J. Davidsen and G. Kwiatek, Phys. Rev. Lett. 110, 068501 (2013).

[31] J. M. Beggs and D. Plenz, J. Neurosci. 23, 11167 (2003).

[32] T. Bellay, A. Klaus, S. Seshadri, and D. Plenz, Elife 4, e07224 (2015).

[33] X. Balandraud, N. Barrera, P. Biscari, M. Grédiac, and G. Zanzotto, Phys. Rev. B 91, 174111 (2015).

[34] S. Field, J. Witt, F. Nori, and X. Ling, Phys. Rev. Lett. 74, 1206 (1995).

[35] E. Altshuler and T. H. Johansen, Rev. Mod. Phys. 76, 471 (2004).

[36] J. P. Sethna, K. A. Dahmen, and C. R. Myers, Nature (London) 410, 242 (2001).

[37] M. Kardar, Phys. Rep. 301, 85 (1998).

[38] D. S. Fisher, Phys. Rep. 301, 113 (1998).

[39] P. Chauve, P. L. Doussal, and K. J. Wiese, Phys. Rev. Lett. 86, 1785 (2001).

[40] A. Rosso, P. Le Doussal, and K. J. Wiese, Phys. Rev. B 80, 144204 (2009).

[41] A. Dobrinevski, P. L. Doussal, and K. J. Wiese, Europhys. Lett. 108, 66002 (2015).

[42] T. Thiery, P. L. Doussal, and K. J. Wiese, J. Stat. Mech. (2015) P08019.

[43] T. Thiery, P. Le Doussal, and K. J. Wiese, Phys. Rev. E 94, 012110 (2016).

[44] M. A. Lebyodkin, I. V. Shashkov, T. A. Lebedkina, and V. S. Gornakov, Phys. Rev. E 95, 032910 (2017).

[45] J. Barés, A. Dubois, L. Hattali, D. Dalmas, and D. Bonamy, Nat. Commun. 9, 1253 (2018).

[46] L. de Arcangelis, C. Godano, J. R. Grasso, and E. Lippiello, Phys. Rep. 628, 1 (2016).

[47] T. Utsu, J. Faculty Sci., Hokkaido Univ. Ser. 7, Geophys. 3, 197 (1971).

[48] A. Helmstetter, Phys. Rev. Lett. 91, 058501 (2003).

[49] M. Båth, Tectonophysics 2, 483 (1965).

[50] F. Omori, J. College Sci. Imperial Univ. Tokyo 7, 111 (1894).

[51] T. Utsu, J. Faculty Sci., Hokkaido Univ. Ser. 7, Geophys. 3, 379 (1972).

[52] T. Utsu, Y. Ogata, and R. Matsu'ura, J. Phys. Earth 43, 1 (1995).

[53] Y. Ogata, J. Am. Stat. Assoc. 83, 9 (1988).

[54] A. Abed Zadeh, J. Barés, J. E. S. Socolar, and R. P. Behringer, Phys. Rev. E 99, 052902 (2019).

[55] J. Barés and D. Bonamy, Philos. Trans. R. Soc. A 377, 20170386 (2018).

[56] R. Sánchez, D. E. Newman, and B. A. Carreras, Phys. Rev. Lett. 88, 068302 (2002).

[57] E. A. Jagla, F. P. Landes, and A. Rosso, Phys. Rev. Lett. 112, 174301 (2014).

[58] E. A. Jagla and A. B. Kolton, J. Geophys. Res.: Solid Earth 115, B05312 (2010).
[59] L. E. Aragón, E. A. Jagla, and A. Rosso, Phys. Rev. E 85, 046112 (2012).

[60] E. E. Ferrero, L. Foini, T. Giamarchi, A. B. Kolton, and A. Rosso, Phys. Rev. Lett. 118, 147208 (2017).

[61] L. Laurson, X. Illa, and M. J. Alava, J. Stat. Mech. (2009) P01019.

[62] F. Font-Clos, G. Pruessner, N. R. Moloney, and A. Deluca, New J. Phys. 17, 043066 (2015).

[63] S. Janićević, L. Laurson, K. J. Måløy, S. Santucci, and M. J. Alava, Phys. Rev. Lett. 117, 230601 (2016).

[64] J. Schmittbuhl, S. Roux, J. P. Vilotte, and K. J. Måløy, Phys. Rev. Lett. 74, 1787 (1995).

[65] S. Ramanathan, D. Ertas, and D. S. Fisher, Phys. Rev. Lett. 79, 873 (1997).

[66] D. Bonamy, S. Santucci, and L. Ponson, Phys. Rev. Lett. 101, 045501 (2008).

[67] J. Barés, M. Barlet, C. L. Rountree, L. Barbier, and D. Bonamy, Front. Phys. 2, 70 (2014).

[68] D. Bonamy, C. R. Phys. 18, 297 (2017).

[69] W. Weibull, Proc. R. Swed. Inst. Eng. Res. 1939, 151 (1939).

[70] B. Lawn, Fracture of Brittle Solids (Cambridge Solid State Science, Cambridge, UK, 1993).

[71] A. A. Griffith, Philos. Trans. R. Soc. London, Ser. A 221, 163 (1921).

[72] L. B. Freund, Dynamic Fracture Mechanics (Cambridge University Press, Cambridge, UK, 1990).

[73] A. B. Movchan, H. Gao, and J. R. Willis, Int. J. Solids Struct. 35, 3419 (1998).

[74] J. R. Rice, J. Appl. Mech. 52, 571 (1985).

[75] H. Gao and J. Rice, J. Appl. Mech. 56, 828 (1989).

[76] J. Barés, L. Barbier, and D. Bonamy, Phys. Rev. Lett. 111, 054301 (2013).

[77] See Supplemental Material at http://link.aps.org/supplemental/ 10.1103/PhysRevE.100.023001 for the jerky motion of a crack front (in red) obtained via simulations.

[78] T. V. Stiphout, J. Zhuang, and D. Marsan, Seismicity declustering, in Community Online Resource for Statistical Seismicity Analysis (2012), doi: 10.5078/corssa-52382934.

[79] M. Stojanova, S. Santucci, L. Vanel, and O. Ramos, Phys. Rev. Lett. 112, 115502 (2014).

[80] O. Duemmer and W. Krauth, J. Stat. Mech. (2007) P01019.

[81] This is generic to the depinning transition where the probability density function of the distances from instability threshold does not vanish at origin. Then the most instable among $L$ elementary blocks always scales as $1 / L$.

[82] J. Barés, Ph.D. thesis, Ecole doctorale de l'X, 2013.

[83] G. Durin and S. Zapperi, Phys. Rev. Lett. 84, 4705 (2000).

[84] S. Santucci, R. Planet, K. J. Måløy, and J. Ortín, Europhys. Lett. 94, 46005 (2011).

[85] X. Clotet, S. Santucci, and J. Ortín, Phys. Rev. E 93, 012150 (2016).

[86] S. Patinet, D. Bonamy, and L. Proville, Phys. Rev. B 84, 174101 (2011).

[87] M. Myllys, J. Maunuksela, M. Alava, T. Ala-Nissila, J. Merikoski, and J. Timonen, Phys. Rev. E 64, 036101 (2001).

[88] T. A. Lebedkina, D. A. Zhemchuzhnikova, and M. A. Lebyodkin, Phys. Rev. E 97, 013001 (2018).

[89] J. Lin, E. Lerner, A. Rosso, and M. Wyart, Proc. Natl. Acad. Sci. USA 111, 14382 (2014). 